\title{
Jet conversions in a quark-gluon plasma
}

\author{
W. Liu, C. M. Ko, and B. W. Zhang* \\ Cyclotron Institute and Physics Department, Texas A\&M University, College Station, Texas 77843-3366, USA
}

(Received 27 July 2006; published 4 May 2007)

\begin{abstract}
Quark and gluon jets traversing through a quark-gluon plasma not only lose their energies but also can undergo flavor conversions. The conversion rates via the elastic $q(\bar{q}) g \rightarrow g q(\bar{q})$ and the inelastic $q \bar{q} \leftrightarrow g g$ scatterings are evaluated in the lowest order in QCD. Including both jet energy loss and conversions in the expanding quark-gluon plasma produced in relativistic heavy ion collisions, we have found a net of quark jets to gluon jets. This reduces the difference between the nuclear modification factors for quark and gluon jets in central heavy ion collisions and thus enhances the $p / \pi^{+}$and $\bar{p} / \pi^{-}$ratios at high transverse momentum. However, a much larger net quark-to-gluon jet conversion rate than the one given by the lowest order QCD is needed to account for the observed similar ratios in central $\mathrm{Au}+\mathrm{Au}$ and $p+p$ collisions at the same energy. Implications of our results are discussed.
\end{abstract}

DOI: 10.1103/PhysRevC.75.051901

PACS number(s): 12.38.Mh, 25.75.-q

One of the most interesting observations in central heavy ion collisions at the Relativistic Heavy Ion Collider (RHIC) is the suppressed production of hadrons with large transverse momentum [1,2]. This phenomenon has been attributed to the radiative energy loss of quark and gluon jets, produced from initial hard scattering of incoming nucleons, as they traverse through produced quark-gluon plasma (QGP) [3-5]. Recent studies have shown that elastic scattering of quark and gluon jets in the QGP also leads to an appreciable loss of their energies [6,7]. Because of its larger color charge, a gluon jet is expected to lose more energy than quark and antiquark jets. Since the ratio of high-momentum protons and antiprotons to pions produced from the fragmentation of a gluon jet is much larger than that from a quark jet, and there are more gluon than quark jets in proton-proton collisions, a larger gluon than quark energy loss would lead to smaller $p / \pi^{+}$and $\bar{p} / \pi^{-}$ratios at high transverse momentum in central heavy ion collisions than in proton-proton collisions at the same energy [8]. This is in contrast to $p / \pi^{+}$and $\bar{p} / \pi^{-}$ ratios at intermediate transverse momentum where they are enhanced in central heavy ion collisions as a result of quark coalescence or recombination [9-11]. Experimentally, data from the STAR Collaboration have indicated, however, that $p / \pi^{+}$and $\bar{p} / \pi^{-}$ratios at high transverse momentum in central $\mathrm{Au}+\mathrm{Au}$ collisions [12] approach those in $p+p$ and $d+\mathrm{Au}$ collisions [13]. This observation implies that the ratio of final quark and gluon jets at high transverse momentum is similar to that of initial ones. A possible mechanism for reducing the effect due to different quark and gluon jet energy losses in QGP is to allow conversions between quark and gluon jets via both elastic $g q(\bar{q}) \rightarrow q(\bar{q}) g$ and inelastic $q \bar{q} \leftrightarrow g g$ scatterings with thermal quarks and gluons in the QGP. The idea of a quark jet converting to a gluon jet has previously been considered in Refs. [14] for deeply inelastic scattering. In the present paper, we study this effect in the lowest order in QCD. We find that conversions between quark and gluon jets indeed lead to an

\footnotetext{
*On leave from Institute of Particle Physics, Huazhong Normal University, Wuhan 430079, China.
}

increase in the final number of gluon jets in central heavy ion collisions than in the case without conversions, but the effect is not large enough to explain the experimental observation. To bring the resulting $p / \pi^{+}$and $\bar{p} / \pi^{-}$ratios at high transverse momentum to the values observed in $p+p$ collisions at the same energy requires a factor of 4 or larger in the increase of the net quark-to-gluon jet conversion rate obtained from the lowest order QCD.

Jet conversion rates in quark-gluon plasma. The conversion rate of a quark jet to a gluon jet or vice versa due to two-body scattering with quarks and gluons in a QGP is given by the collisional width

$$
\begin{aligned}
\Gamma_{C}= & \frac{1}{2 E_{1}} \int \frac{g_{2} d^{3} \mathbf{p}_{2}}{(2 \pi)^{3} 2 E_{2}} \frac{d^{3} \mathbf{p}_{3}}{(2 \pi)^{3} 2 E_{3}} \frac{d^{3} \mathbf{p}_{4}}{(2 \pi)^{3} 2 E_{4}} \\
& \times f\left(\mathbf{p}_{2}\right)\left[1 \pm f\left(\mathbf{p}_{3}\right)\right]\left[1 \pm f\left(\mathbf{p}_{4}\right)\right] \overline{\left|\mathcal{M}_{12 \rightarrow 34}\right|^{2}} \\
& \times(2 \pi)^{4} \delta^{(4)}\left(p_{1}+p_{2}-p_{3}-p_{4}\right)
\end{aligned}
$$

divided by $\hbar$. In the above, $E_{1}$ and $E_{3}$ are, respectively, energies of the jet before and after conversion, while $E_{2}$ and $E_{4}$ are those of quarks and gluons with degeneracy $g_{2}$ in the QGP; $f(\mathbf{p})$ is the thermal distribution with + and - referring to gluons and quarks, respectively; and $\overline{\left|\mathcal{M}_{12 \rightarrow 34}\right|^{2}}$ is the squared scattering amplitude after averaging over the spins and colors of initial partons and summing over those of final partons and is well known for both the elastic scattering $q(\bar{q}) g \rightarrow$ $g q(\bar{q})$ and the inelastic scattering $q \bar{q} \leftrightarrow g g$. To ensure that the quark (gluon) jet is converted to a gluon (quark) jet in elastic scattering, the gluon (quark) in the final state is required to have a larger momentum. Since both elastic and inelastic scattering are dominated by forward-peaked $t$ - and backward-peaked $u$-channel diagrams, the momentum of the converted jet is very close to that of the initial jet. Neglecting the effect due to the soft parton in a conversion process is thus justified.

To take into account medium effects, we include the thermal masses $m_{q}=m_{g} / \sqrt{3}=g T / \sqrt{6}$ [15] for quarks and gluons in a QGP at temperature $T$, where $g$ is the QCD coupling constant. With $\alpha_{s}=g^{2} / 4 \pi=0.3$, appropriate for energy scales considered here, calculated collisional widths for gluonto-quark (upper panels) and quark-to-gluon (lower panels) jet 


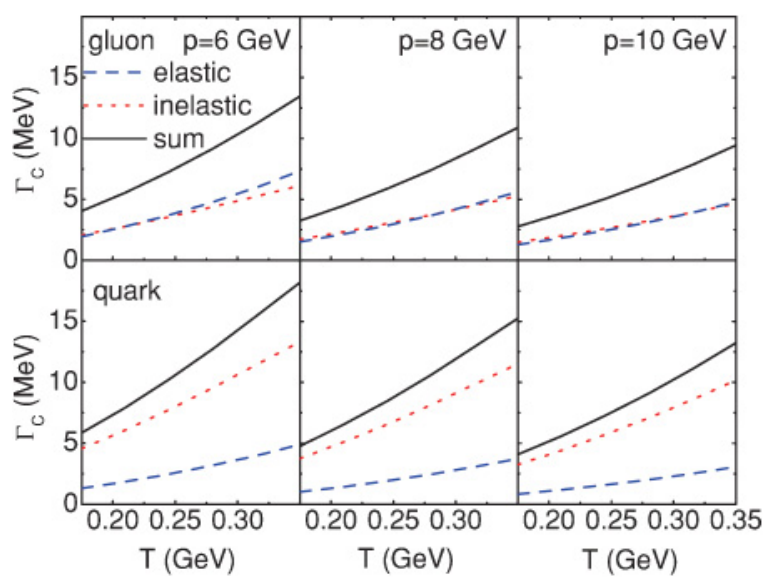

FIG. 1. (Color online) Collisional widths for gluon-to-quark (upper panels) and quark-to-gluon (lower panels) jet conversions in QGP due to elastic scattering $q(\bar{q}) g \rightarrow g q(\bar{q})$ and inelastic scattering $q \bar{q} \leftrightarrow g g$ as well as their sum, as functions of temperature for different quark and gluon momenta $p$ in $\mathrm{GeV} / c$.

conversions in a chemically equilibrated QGP are shown in Fig. 1 for jets of momentum 6,8 , and $10 \mathrm{GeV} / c$. Because of larger (about a factor of 2) quark than gluon densities in the chemically equilibrated QGP with thermal quark and gluon masses, contributions from elastic (dashed lines) and inelastic (dotted lines) scattering to conversion of gluon jets to quark jets are comparable, while inelastic scattering is more important than elastic scattering for quark-to-gluon jet conversion. Adding both contributions leads to a larger total conversion rate for the quark jets than for the gluon jets, particularly at high transverse momentum, as shown by solid lines in Fig. 1.

Drag coefficients for jets in quark-gluon plasma. As in our study of heavy quark energy loss [16], the momentum degradation of a quark or gluon jet in the QGP depends on its drag coefficient, which is given by averages similar to that for the collisional width, i.e.,

$$
\gamma(|\mathbf{p}|, T)=\sum_{i}\left\langle\overline{\left|\mathcal{M}_{i}\right|^{2}}\right\rangle-\sum_{i}\left\langle\overline{\left|\mathcal{M}_{i}\right|^{2}} \mathbf{p} \cdot \mathbf{p}^{\prime}\right\rangle /|\mathbf{p}|^{2},
$$

where $\mathbf{p}$ and $\mathbf{p}^{\prime}$ are the momenta of the jet before and after a collision, respectively, and the sum is over all scattering processes. Since the present study is mainly concerned with conversions between gluon and quark jets in the QGP, we only consider explicitly the contribution of two-body scattering to their energy losses and mimic the effect of the more important radiative energy loss by introducing a phenomenological multiplication factor $K_{E}$.

In Fig. 2, we show the gluon and quark drag coefficients $\gamma_{N}$ due to nonconversion (upper panels) and $\gamma_{C}$ due to conversion (lower panels) scatterings as functions of their momentum in a QGP at temperatures $T=300$ or $T=200 \mathrm{MeV}$. It is seen that the drag coefficients due to nonconversion scattering are much larger than those due to conversion scattering. While the drag coefficient for the gluon jet is about a factor of 2 larger than that for the quark jet in nonconversion scattering, they are similar in conversion scattering. Enhancing the calculated drag coefficients due to two-body scattering by a factor $K_{E}=4$,

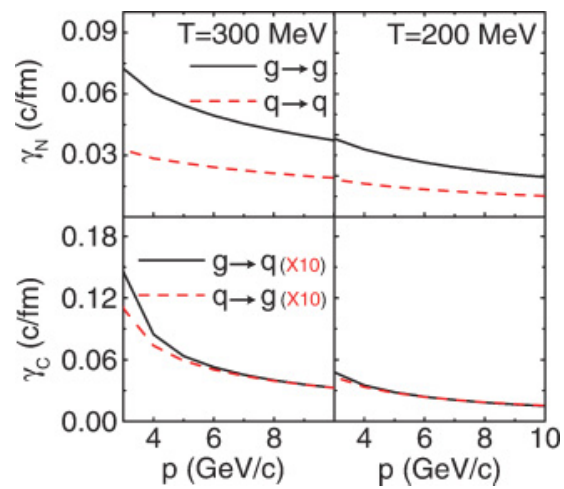

FIG. 2. (Color online) Drag coefficients for gluon and quark jets in QGP due to nonconversion (upper panels) and conversion (lower panels) two-body scattering as functions of their momentum in QGP at temperature $T=300$ or $200 \mathrm{MeV}$.

we find that both their values and transverse momentum dependence are consistent with those extracted from the energy loss formula derived in Ref. [4] for radiative energy losses of quark and gluon jets in a quark-gluon plasma. We have also checked that with such enhanced drag coefficients, the observed quenching of high transverse momentum pions at RHIC can indeed be described.

Initial jet distributions and heavy ion collision dynamics. To see the effect of conversions between quark and gluon jets on their energy losses in QGP, we consider central Au+Au collisions at center-of-mass energy $\sqrt{s_{N N}}=200 \mathrm{GeV}$. The initial transverse momentum spectra of quark, antiquark, and gluon jets at midrapidity are obtained by multiplying those from PYTHIA for $p+p$ collisions at the same energy by the number of binary collisions $(\sim 960)$ in central $\mathrm{Au}+\mathrm{Au}$ collisions. The resulting transverse momentum spectra of initial partonic jets can be parametrized by

$$
\frac{d N_{i}}{d^{2} p_{T}} \approx A_{i} /\left(1+p_{T} / B_{i}\right)^{\alpha_{i}},
$$

with $i=q, \bar{q}$, and $g$. With transverse momentum $p_{T}$ in units of $\mathrm{GeV} / c$, values of the parameters $A_{i}, B_{i}$, and $\alpha_{i}$ in Eq. (3) are given in Table I.

For the dynamics of formed QGP, we follow that of Ref. [17] by assuming that it evolves boost invariantly in the longitudinal direction but with an accelerated transverse expansion. Specifically, its volume expands in the proper time $\tau$ according to $V(\tau)=\pi R(\tau)^{2} \tau c$, where $R(\tau)=$ $R_{0}+a\left(\tau-\tau_{0}\right)^{2} / 2$ is the transverse radius with an initial value $R_{0}=7 \mathrm{fm}, \tau_{0}=0.6 \mathrm{fm} / c$ is the QGP formation time, and $a=0.1 \quad c^{2} / \mathrm{fm}$ is the transverse acceleration. Starting with

TABLE I. Parameters for initial minijet parton distributions given in Eq.(3) at midrapidity from $\mathrm{Au}+\mathrm{Au}$ at $\sqrt{s_{N N}}=200 \mathrm{GeV}$.

\begin{tabular}{cccc}
\hline \hline & $A\left[(\mathrm{GeV} / \mathrm{c})^{-2}\right]$ & $B(\mathrm{GeV} / c)$ & $\alpha$ \\
\hline$g$ & 1440 & 1.5 & 8.0 \\
$q$ & 670 & 1.6 & 7.9 \\
$\bar{q}$ & 190 & 1.9 & 8.9 \\
\hline \hline
\end{tabular}


an initial temperature $T_{i}=350 \mathrm{MeV}$, time dependence of the temperature is obtained from entropy conservation, leading to the critical temperature $T_{c}=175 \mathrm{MeV}$ at the proper time $\tau_{c}=5 \mathrm{fm} / c$.

Jet energy loss in quark-gluon plasma. For a quark or gluon jet moving through the QGP, the rate for the change of its mean transverse momentum $\left\langle p_{T}\right\rangle$ is given by

$$
\frac{d\left\langle p_{T}\right\rangle}{d \tau}=-\left\langle\gamma\left(p_{T}, T\right) p_{T}\right\rangle \approx-\gamma\left(\left\langle p_{T}\right\rangle, T\right)\left\langle p_{T}\right\rangle .
$$

In obtaining the last expression of this equation, we neglected the dispersion of the jet momentum during its propagation and thus assumed that $\left\langle p_{T}^{2}\right\rangle \approx\left\langle p_{T}\right\rangle^{2}$. This is expected to be a reasonable approximation for high transverse momentum particles.

Because of conversion scatterings, a quark or gluon jet can be converted to a gluon or quark jet with a rate given by the corresponding collisional width. These effects are modeled by introducing a large number of test quark and gluon jets that are distributed in the transverse plane according to that of underlying binary nucleon-nucleon collisions. Their transverse momentum distribution is taken to be uniform with directions pointed isotropically in the transverse plane. A test jet of a given kind with certain transverse momentum is assigned a probability that is proportional to the corresponding jet momentum spectrum with the proportional constant determined by requiring that the sum of the probabilities for all test jets of this kind is equal to their total number. Motions of the jets are then followed via straight trajectories. After a small time step $\Delta \tau$, which is usually taken to be $0.1 \mathrm{fm} / c$, a random number $x$ is chosen and compared with $y=\Gamma_{C} \Delta \tau / \hbar$. If $x$ is smaller than $y$, then the jet is converted to a different kind with a reduction of its momentum $p_{T}$ by $\gamma_{C} \Delta \tau p_{T}$ but without changing its associated probability. Otherwise, the jet loses a momentum $\gamma_{N} \Delta \tau p_{T}$. This sequence of processes is repeated until the smaller of the time when the QGP phase ends and the time for the jet to escape the expanding QGP. The final momentum distributions of quark and gluon jets are obtained from those of test jets by multiplying with their associated probabilities.

Results. The ratio of final quark or gluon jet spectrum to its initial spectrum, defined as its nuclear modification factor $R_{A A}$, is shown in Fig. 3. Upper and lower dash-dotted lines are those for the quark and gluon jets using drag coefficients from two-body elastic scattering that are enhanced by the factor $K_{E}=4$ but without jet conversions. The smaller $R_{A A}$ for gluons than for quarks is a result of their larger energy loss. Although final high transverse momentum quark and gluon jets are initially produced near the surface of the QGP fireball, quark jets tend to originate from a region that is deeper inside the fireball than that for gluon jets as a result of their smaller drag coefficients. With the larger quark-to-gluon conversion width than that for gluon-to-quark conversion, it is thus more likely for quark jets to convert to gluon jets than for gluon jets to convert to quark jets. Including conversions between quark and gluon jets through conversion scatterings thus reduces the difference between the quark and gluon $R_{A A}$ as shown by dotted lines in Fig. 3.

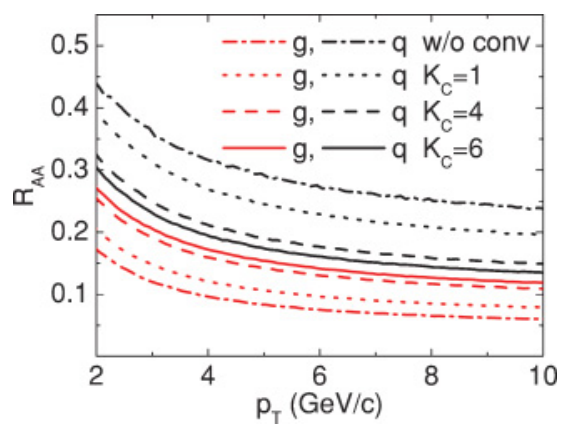

FIG. 3. (Color online) Nuclear modification factors for quark (upper lines) and gluon (lower lines) jets in central $\mathrm{Au}+\mathrm{Au}$ collisions at $\sqrt{s_{N N}}=200 \mathrm{GeV}$ as functions of momentum without or with different enhancement factors $K_{C}$ for conversion scattering.

To obtain the proton, antiproton, and pion spectra at high transverse momentum from those of quark and gluon jets, we use the AKK fragmentation function [18], which has been shown to reproduce measured charged pion, proton, and antiproton spectra at high transverse momentum in $p+p$ and $d+\mathrm{Au}$ collisions at $\sqrt{s}_{N N}=200 \mathrm{GeV}$. In particular, the resulting $\bar{p} / p$ ratio at high transverse momentum in $p+p$ collisions is about 0.7 and agrees with the observed value. While protons and antiprotons are equally produced from gluon fragmentation, fragmentation of quark and antiquark jets is known to produce mainly protons and antiprotons, respectively $[12,13,19]$. We therefore assume that no antiprotons are produced from a quark jet and no protons are produced from an antiquark jet as in Ref. [13].

Results for $p / \pi^{+}$and $\bar{p} / \pi^{-}$ratios in central $\mathrm{Au}+\mathrm{Au}$ collisions at $\sqrt{s_{N N}}=200 \mathrm{GeV}$ are shown in Fig. 4. Ratios for the case without conversions between quark and gluon jets, are significantly smaller than those in $p+p$ collisions. Including conversions between quark and gluon jets increases the $p / \pi^{+}$and $\bar{p} / \pi^{-}$ratios, but they are still below those from $p+p$ collisions.

The $p / \pi^{+}$and $\bar{p} / \pi^{-}$ratios are increased or the difference between the quark and gluon $R_{A A}$ is reduced if the conversion

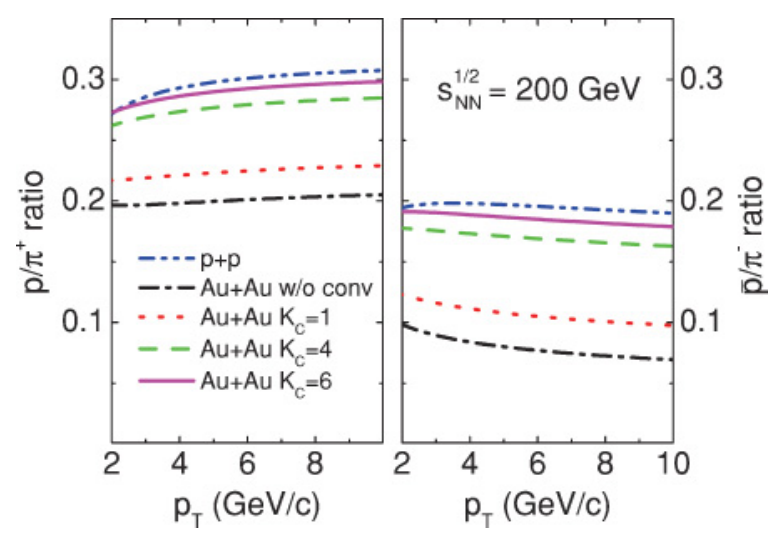

FIG. 4. (Color online) $p / \pi^{+}$(left panel) and $\bar{p} / \pi^{-}$(right panel) ratios from quark and gluon jet fragmentation in central $\mathrm{Au}+\mathrm{Au}$ collisions at $\sqrt{s_{N N}}=200 \mathrm{GeV}$ as functions of momentum without or with different enhancement factors $K_{C}$ for conversion scattering. Ratios are also shown for $p+p$ collisions at same energy. 
widths shown in Fig. 1 are enhanced by a factor $K_{C}$. As shown in Figs. 3 and 4, with $K_{C}=4$, similar to the enhancement factor $K_{E}$ for quark and gluon drag coefficients, and larger, e.g., $K_{C}=6$, the quark and gluon nuclear modification factors become closer, and the final $p / \pi^{+}$and $\bar{p} / \pi^{-}$ratios are also more similar to those in $p+p$ collisions.

We note that quark and gluon jet conversions have a larger effect on the $\bar{p} / \pi^{-}$ratio than the $p / \pi^{+}$ratio. This is because of the smaller number of antiquark jets than quark jets in heavy ion collisions and the assumption that quark and antiquark jets fragment only to protons and antiprotons, respectively. Including some admixture of proton and antiproton production from quark jets as well as from antiquark jets would reduce the difference in the jet conversion effect on $p / \pi^{+}$and $\bar{p} / \pi^{-}$ ratios.

As to the effect of using different fragmentation functions, we do not expect them to change our conclusions. For example, if we use instead the KKP fragmentation function [20], which describes the pion spectrum well but underestimates the proton and antiproton spectra at high transverse momentum by about a factor of 3 , jet conversions are still needed to obtain the same $p / \pi^{+}$and $\bar{p} / \pi^{-}$ratios in both cental $\mathrm{Au}+\mathrm{Au}$ collisions and $p+p$ collisions at the same energy. Of course, their values are about a factor of 3 smaller than measured ones.

Discussion and conclusions. We have studied the effect of both elastic and inelastic two-body scatterings of quark and gluon jets in a quark-gluon plasma not only on their energy loss but also on the conversions between them. Although inelastic two-body scatterings of quark-antiquark annihilation and gluon-gluon fusion always lead to conversions between quark and gluon jets, elastic two-body scatterings can be nonconversion scatterings if the momentum of the quark or gluon jet remains to be the one with a larger transverse momentum after the scattering. To mimic the effect of radiative energy loss of quark and gluon jets in the quark-gluon plasma, we multiplied the drag coefficients calculated from two-body scattering by a factor of 4 . We found that two-body conversion scatterings lead to a small net conversion of the quark jets to the gluon jets, resulting in slightly larger $p / \pi^{+}$and $\bar{p} / \pi^{-}$ ratios than in the case without including conversions between gluon and quark jets, but the conversion is not large enough to bring these ratios in central $\mathrm{Au}+\mathrm{Au}$ collisions to those in $p+p$ collisions at the same energy. A large conversion enhancement factor of more than 4 , similar to that needed for the jet drag coefficients to describe the jet energy loss, is needed to explain the experimental observations. We note that somewhat smaller enhancement factors would be needed if the time for starting the interactions of initial jets with the QGP is earlier, such as the formation time $\sim 1 / p_{T}$ of the jets, instead of $0.5 \mathrm{fm} / c$ used in the present study. For example, with a starting time of $0.05 \mathrm{fm} / c$ reduces the enhancement factors by about $20 \%$.
In our study, we assumed that the QGP produced at RHIC is in chemical equilibrium with about twice as many quarks and antiquarks than gluons. If the produced partonic matter is a pure gluon matter, the resulting $p / \pi^{+}$and $\bar{p} / \pi^{-}$ratios in central $\mathrm{Au}+\mathrm{Au}$ collisions turn out to be slightly smaller than those from a chemically equilibrated QGP as the gluon matter enhances the conversion of gluon jets to quark jets via the inelastic scattering $g g \rightarrow q \bar{q}$. On the other hand, pure quark and antiquark matter gives slightly larger $p / \pi^{+}$and $\bar{p} / \pi^{-}$ ratios than from a chemically equilibrated QGP as the rate for the inelastic conversion process $q \bar{q} \rightarrow g g$ is enhanced. As for a chemically equilibrated QGP, none of these two scenarios is able to increase the $p / \pi^{+}$and $\bar{p} / \pi^{-}$ratios at high transverse momentum in central $\mathrm{Au}+\mathrm{Au}$ collisions to approach those in $p+p$ collisions at the same energy without requiring a large enhancement factor for the net quark-to-gluon jet conversion rate.

Our results that the net quark-to-gluon jet conversion rate in central heavy ion collisions at RHIC is much larger than that given by the lowest order in QCD may not be surprising, as the jet drag coefficients from the lowest order QCD need to be enhanced by a similar factor to describe their energy losses in QGP. Also, previous studies using the multiphase transport (AMPT) model [21], which includes only two-body scattering among partons, have shown that a much larger parton scattering cross section than that given by the lowest order QCD is needed to describe many other experimental observations at RHIC such as the the large elliptic flows of hadrons made of light quarks [22,23] or heavy quarks [24] and the two-pion correlation functions [25]. The large enhancement factor over the lowest order QCD results can be considered as an effective parameter for taking into account effects not considered in present study such as higher order contributions and multibody scattering. A preliminary study of jet conversions via higher order radiative scatterings with thermal partons shows that they lead to similar quark and gluon jet conversion widths as for the lowest order two-body scatterings considered in the present study. Also, using the QCD coupling constant at finite temperature as extracted from the lattice QCD studies [26], which is about a factor of 2 larger than that from the perturbative QCD, further increases the quark and gluon jet conversion widths, resulting in a conversion enhancement factor close to the needed one. Our results thus could be another indication for the strongly coupled QGP that has been produced in central heavy ion collisions at RHIC [27].

We thank Zhangbu $\mathrm{Xu}$ and Carl Gagliardi for helpful discussions. This work was supported in part by the U.S. National Science Foundation under Grant No. PHY-0457265, and the Welch Foundation under Grant No. A-1358. The work of B.W.Z. was further supported by the National Natural Science Foundation of China under Project No. 10405011.
[1] PHENIX Collaboration, A. Adcox et al., Phys. Rev. Lett. 88, 022301 (2002).

[2] C. Adler et al. (STAR Collaboration), Phys. Rev. Lett. 89, 202301 (2003); 90, 082302 (2003).
[3] X. N. Wang, Phys. Lett. B579, 299 (2004).

[4] M. Gyulassy, P. Lévai, and I. Vitev, Phys. Rev. Lett. 85, 5535 (2001); Nucl. Phys. B594, 371 (2001).

[5] U. A. Wiedemann, Nucl. Phys. B588, 303 (2000). 
[6] M. G. Mustafa, Phys. Rev. C 72, 014905 (2005); M. G. Mustafa and M. H. Thoma, Acta Phys. Hung. A 22, 93 (2005).

[7] S. Wicks, W. Horowitz, M. Djordjevic, and M. Gyulassy, Nucl. Phys. A784, 426 (2007).

[8] X. N. Wang, Phys. Rev. C 58, 2321 (1998).

[9] R. C. Hwa and C. B. Yang, Phys. Rev. C 67, 034902 (2003); 67, 064902 (2003).

[10] V. Greco, C. M. Ko, and P. Lévai, Phys. Rev. Lett. 90, 202302 (2003); Phys. Rev. C 68, 034904 (2003).

[11] R. J. Fries, B. Müller, C. Nonaka, and S. A. Bass, Phys. Rev. Lett. 90, 202303 (2003); Phys. Rev. C 68, 044902 (2003).

[12] J. Adams et al. (STAR Collaboration), Phys. Rev. Lett. 97, 152301 (2006).

[13] J. Adams et al. (STAR Collaboration), Phys. Lett. B637, 161 (2006).

[14] X. N. Wang and X. Guo, Nucl. Phys. A696, 788 (2001); B. W. Zhang, X. N. Wang, and A. Schäfer, ibid. A783, 551c (2007).

[15] J. P. Blaizot and E. Iancu, Phys. Rep. 359, 355 (2002).

[16] W. Liu and C. M. Ko, Nucl. Phys. A783, 233c (2007); nucl-th/ 0603004
[17] L. W. Chen, V. Greco, C. M. Ko, S. H. Lee, and W. Liu, Phys. Lett. B601, 34 (2004).

[18] S. Albino, B. A. Kniehl, and G. Kramer, Nucl. Phys. B725, 181 (2005).

[19] P. B. Straub et al., Phys. Rev. D 45, 3030 (1992).

[20] B. A. Kniehl, G. Kramer, and B. Pötter, Nucl. Phys. B597, 337 (2001).

[21] Z. W. Lin, C. M. Ko, B. A. Li, B. Zhang, and S. Pal, Phys. Rev. C 72, 064901 (2005).

[22] Z. W. Lin and C. M. Ko, Phys. Rev. C 65, 034904 (2002).

[23] L. W. Chen, C. M. Ko, and Z. W. Lin, Phys. Rev. C 69, 031901(R) (2004).

[24] B. Zhang, L. W. Chen, and C. M. Ko, Phys. Rev. C 72, 024906 (2005).

[25] Z. W. Lin, C. M. Ko, and S. Pal, Phys. Rev. Lett. 89, 152301 (2002).

[26] O. Kaczmarek, F. Karsch, F. Zantow, and P. Petreczky, Phys. Rev. D 70, 074505 (2004).

[27] STAR Collaboration, Nucl. Phys. A757, 102 (2005); PHENIX Collaboration, ibid. A757, 184 (2005) . 